\title{
Branch-coverage testability transformation for unstructured
} programs

\author{
R. M. Hierons ${ }^{1}$, M. Harman ${ }^{2}$ and C. J. Fox ${ }^{3}$ \\ ${ }^{1}$ Department of Information Systems and Computing, Brunel University, Uxbridge, \\ Middlesex, UB8 3PH, United Kingdom \\ ${ }^{2}$ Department of Computer Science, King's College London, Strand, London, WC2R 2LS, \\ United Kingdom. \\ ${ }^{3}$ Department of Computer Science, University of Essex, Colchester CO4 3SQ, United \\ Kingdom \\ Email: rob.hierons@brunel.ac.uk
}

\begin{abstract}
Generating test data by hand is a tedious, expensive and error-prone activity, yet testing is a vital part of the development process. Several techniques have been proposed to automate the generation of test data, but all of these are hindered by the presence of unstructured control flow. This paper addresses the problem using testability transformation. Testability transformation does not preserve the traditional meaning of the program, rather it is concerned with preserving the test adequacy of sets of input data. This requires new equivalence relations, which, in turn, entails novel proof obligations. The paper illustrates this using the branch coverage adequacy criterion and develops a branch adequacy equivalence relation and a testability transformation for restructuring. It then presents a proof that the transformation is branch adequacy preserving.
\end{abstract}

Keywords: branch coverage, transformation, testability, exit statements

Received month date, year; revised month date, year; accepted month date, year

\section{INTRODUCTION}

Testing is an important part of the software verification process. However, it is also an expensive process, often taking up in the order of fifty percent of the cost of development [1].

When generating tests, it is normal to use a test criterion that states what it means for a set of test inputs to be sufficient (or 'adequate' in the nomenclature of software testing). There are two main classes of test criteria: white-box criteria, which are based on the structure of the code, and black-box criteria, which are based on the specification. Given a test criterion, the problem then is to find test data that satisfies this criterion.

Producing test data by hand is tedious, expensive and error-prone. For these reasons, automated test-data generation has remained a topic of interest for the past three decades. Several techniques for automated testdata generation have been proposed, including symbolic execution $[2,3,4]$, constraint solving $[5,6]$ and searchbased testing $[7,8,9,10,11,12,13,14]$.

Unstructured programs present a problem for all of these automated test-data generation techniques. In this paper, an unstructured program will be defined as one which contains any form of explicit jump statement, typically represented by goto, exit, break or continue statements. This is to be contrasted with a structured program, in which the only control flow that is permitted is that which is expressed by if-then-else-fi conditionals, single-exit while-do-od loops, and statement sequencing.

Early approaches $[2,4]$ to test-data generation used symbolic execution. The symbolic execution technique seeks to symbolically execute the program to allow the test data generator to express constraints on predicates to be executed in terms of initial state variables.

In the 1990s, constraint-solving techniques were developed $[15,5]$. The constraint-solving technique is, in essence, a mirror image of the symbolic execution approach; it seeks to push the constraints on predicates backwards to the initial statement of the program, at which point they become transformed into constraints upon the input of the program.

Both symbolic execution and constraint solving face problems in the presence of loops, because it is difficult to determine a precise transformation to apply to a set of constraints in order to move it through a loop boundary. These problems with loops are further 
compounded if the program to be tested is unstructured as there is then the additional problem of determining where the implicit loop boundaries are, although it is possible to determine the overall control-flow structure [16].

To overcome difficulties caused by loops for constraint-based and symbolic-execution based testdata generation, more recent work has focused upon the use of search-based techniques such as hill climbing [7], simulated annealing [14] and genetic algorithms $[8,9,10,11,12,13]$. These search-based techniques all share the property that they view the space of inputs to the program under test as a search space. The criterion which expresses adequacy of sets of test inputs guides the automated search for such sets.

In the chaining method [7], data flow information is used to locate previous definitions of variables mentioned in a 'problem predicate'. A problem predicate is one for which test data that forces the predicate to select a particular branch has yet to be found. Hill climbing is then applied to attempt to find input values that force the problem predicate to achieve the required value through a given set of definitions of variables mentioned in this predicate. If this fails, a different set of definitions is chosen or the attempt is abandoned. Work on the chaining method has hitherto focussed on achieving full statement coverage but it has been argued that this approach could be extended to other test criteria [7].

In the simulated annealing and genetic algorithms methods, the test criterion is transformed to a fitness function, which guides the search for test data. In the case of branch coverage (and related structural criteria), the fitness function must capture how close a candidate input comes to executing the desired branch. The success of these approaches in finding test data for real systems, has led to them receiving much attention. However, the definition of the fitness function requires control dependence analysis, which is notoriously problematic in the presence of poor structure $[17,18,19,20]$. The presence of many gotos makes it hard to determine how close a test input comes to hitting a desired branch, because the flow of control is not obvious. Consider the program below.

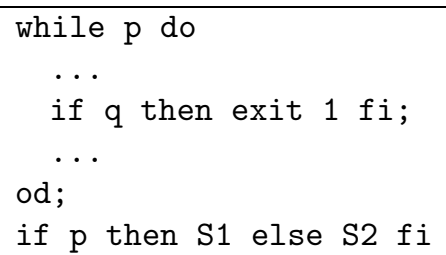

In this program, one target node will be the statement S2 which occurs after the end of the while loop. Notice that there are (apparently) two ways execution can reach $\mathbf{S 2}$. The loop may terminate 'normally', because the predicate $\mathrm{p}$ becomes false, or it may terminate 'abnormally' through the exit statement in the body of the loop. Now, in the case of this program, should the loop terminate normally, then it will be impossible for S2 to be executed, since all paths which involve normal termination and execution of S2 are infeasible. Therefore, the only way in which $\mathrm{S} 2$ could possibly be executed, would be for the loop to terminate through the exit statement.

Unfortunately, the problem of determining which paths are feasible is not decidable. In a test-data generation system, the program will be instrumented to compute values which track the execution of the program. The test-data generator will attempt to force the loop to terminate, either through the exit statement or through 'normal' termination. However, the test-data generator will not (and cannot) know which of the two ways of terminating is more likely to lead to the execution of the desired target node, S2. In extreme cases, such as this one, where one termination route is infeasible, the test-data generator may spend all its effort attempting to generate a test input which drives the computation down an infeasible path.

Even in less extreme cases, it is possible for the testdata generator to waste effort, because two distinct paths must be optimised for, rather than one. If the program is restructured, so that there is only a single way of terminating the loop, then this possibility disappears.

Current techniques and tools for automatically generating test data, to satisfy white-box test criteria, are only applicable to structured code. Thus the applicability of all such tools and techniques will be extended by the development of transformations that take unstructured code and return structured code from which we can generate test data.

Traditional work on transformation of unstructured programs is concerned largely with making the program easier to understand [21], or with compiler optimisations and parallelisations [22]. Of course, the resultant structured programs have other advantageous properties, such as lending themselves to compositional analysis and abstract interpretation, without recourse to continuation based semantics. They also support efficient program dependence graph generation.

In general, the goal of such transformations is to remove goto statements, and create, where possible, a single-entry, single-exit control flow structure. In order to transform unstructured programs to improve test-data generation, it is also important to produce single-entry, single-exit control flow. However, as will be seen, the goal of improving testability, rather than comprehension or compiler optimistations, leads to very different transformation algorithms, equivalence relations and (consequently) proof obligations.

Although there are several kinds of statements that give rise to unstructured programs, this paper will focus upon the exit (or break) statements, which are a common source of unstructuredness. The paper considers the problem of transforming a program $p$ to form a structured program $p^{\prime}$ such that any set of test 
inputs that provides $100 \%$ branch coverage for $p^{\prime}$ also provides $100 \%$ branch coverage for $p$.

In Section 4 we show that programs exists with exit statements that are not path equivalent to any structured program. Thus, we cannot expect any algorithm that removes exit statements to preserve path equivalence. Similarly, it is clear that we need something other than functional equivalence: we need to preserve elements of the structure in order to ensure that the meaning of the adequacy criterion remains unchanged after transformation.

Our approach uses testability transformation [23]. A testability transformation does not need to preserve the traditional meaning of the program to be transformed. Rather it preserves the sets of test inputs which are adequate according to the test criterion. In our case, we are concerned with branch-coverage preserving transformations. Motivated by this, we define a new form of equivalence called branch-coverage equivalence (defined in Section 5). This new form of equivalence gives rise to new transformations and thus new 'proof of correctness' obligations.

We then give a transformation algorithm that takes a program $p$ with multiple-level exit statements ${ }^{4}$ and returns a branch-coverage equivalent program $p^{\prime}$ such that any set $T$ of test inputs that provides $100 \%$ branch coverage for $p^{\prime}$ is guaranteed to give $100 \%$ branch coverage for $p$. A proof of correctness is given in terms of preservation of branch-coverage adequate test data.

The paper is structured as follows. Section 2 describes control-flow graphs and two notions of program equivalence, and restates the notion of a structured program. Section 3 describes exit statements and branch coverage. Section 4 describes related work. Section 5 defines what it means for two programs to be branch-coverage equivalent. Section 6 introduces a transformation algorithm and proves that it is correct. Finally, Section 7 draws conclusions and discusses future work.

\section{PRELIMINARY DEFINITIONS}

\subsection{The syntax}

This section will briefly outline the syntax considered in this paper. We will assume that the problem is to take a single procedure or function that contains one or more exit statements, and transform this into code that contains no exit statements.

This paper will focus on one common construct that leads to unstructured programs: the use of exit statements in loops. An exit statement leads to the flow of control leaving a loop. The presence of an exit thus leads to more than one way in which the flow of control may leave a loop. Some programming languages allow multiple-level exit statements.

\footnotetext{
${ }^{4}$ We assume that each loop has at most one exit statement. We say more about this restriction in Section 6 .
}

The only control structures considered in this paper are while-do-od loops and if-then-else-fi statements. This simplifies the exposition. However, it will become clear that the approach may be extended to other control constructs.

For the purposes of the transformations in this paper, we 'abstract out' all information regarding assignments, input statements, and output statements: these are considered to be atomic statements. Only the control structure is of relevance, as are the exit statements. The syntax is defined in Figure 1.

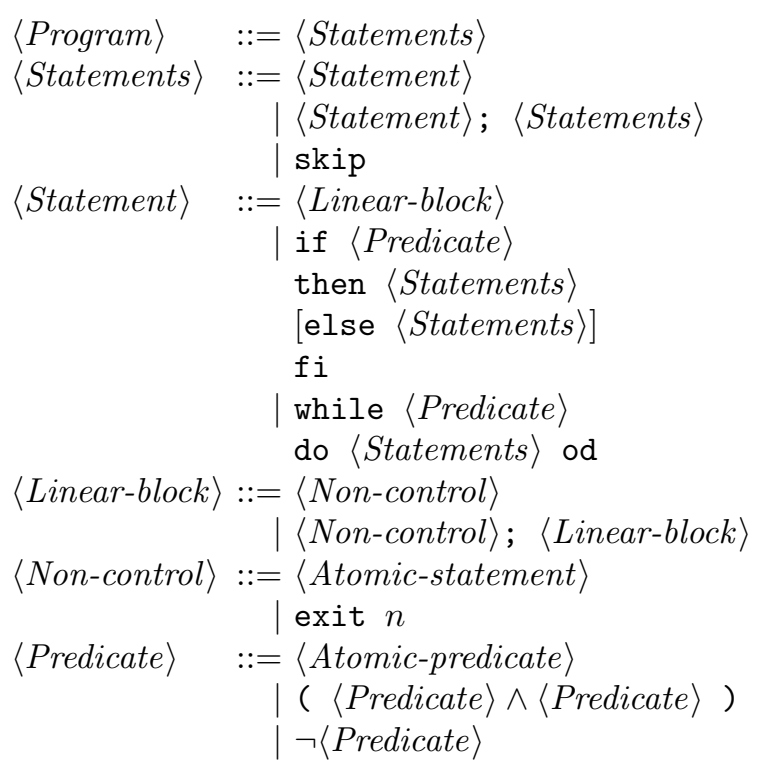

FIGURE 1. Syntax of the Pseudo-Code. For our purposes, it is sufficient to abstract $\langle$ Atomic-statement $\rangle$ and $\langle$ Atomic-predicate $\rangle$ to sets of identifiers. $n$ denotes the natural numbers. Statements of the form exit $n$ must appear at an appropriate depth within nested while loops.

A multiple-level exit statement is parameterised by a non-negative integer whose value is known at compile time. The semantics are defined such that exit $n$ jumps to the statement immediately after the end of the $n^{\text {th }}$ enclosing loop. This paper will use the Wide Spectrum Language (WSL) convention [24] that when $n$ is zero, the meaning of exit $n$ is identical to skip; this convention reduces the number of transformation rules required to cover all cases. Such multiple-level exit statements are sufficiently powerful to capture the full complexity of the unstructuredness issue, since an arbitrary goto program can be converted into a program with multiple-level exits, without the introduction of any new variables [21].

Observe that the process outlined in this paper will be applicable to code that contains many functions and procedures. This is because it is sufficient to consider the individual functions and procedures. However, white-box test criteria, such as $100 \%$ branch coverage, are typically applied in unit testing where individual 
modules or classes are tested.

\subsection{The Control Flow Graph}

Given a program $p$, the control flow graph (CFG) for $p$ is a directed graph $G$ that has a set of vertices that represent the statements and predicates of $p$ and edges between these vertices. The edges are defined by rules that aim to represent the possible flow of control. For example, in the part of the CFG that represents

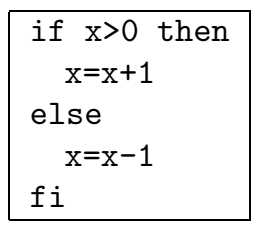

if the predicate $\mathrm{x}>0$ is represented by vertex $n_{1}, \mathrm{x}=\mathrm{x}+1$ is represented by vertex $n_{2}$ and $\mathrm{x}=\mathrm{x}-1$ is represented by vertex $n_{3}$ then there is an edge from $n_{1}$ to $n_{2}$ and an edge from $n_{1}$ to $n_{3}$. In this case, there will also be exit edges from vertices $n_{2}$ and $n_{3}$ that merge, indicating a common flow of control following this statement. This is illustrated in Figure 2.

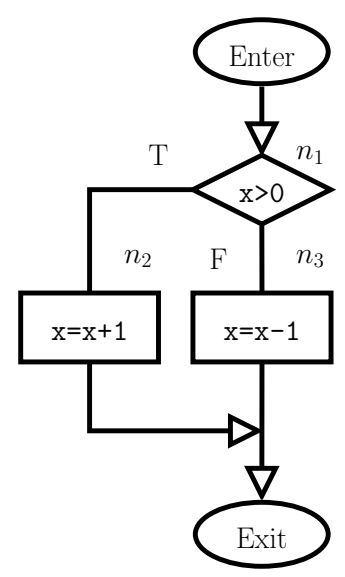

FIGURE 2. Control Flow Graph for the program fragment if $x>0$ then $x=x+1$ else $x=x-1$ fi

The CFG has two special vertices: the start vertex that represents the start of execution (or "entrance" into the block of code represented by the CFG) and the end vertex that represents termination (or "exit" from the code block represented by the CFG).

Note that the edges are defined using rules that abstract away certain details. Thus, there may be edges in the CFG that cannot be traversed. For example, the CFG for the code

$x=0 ;$
if $x>0$ then
$\quad x=x+1$
fi

has an edge to the vertex representing $\mathrm{x}=\mathrm{x}+1$ even though this statement is not reachable.

At times we will have to talk about paths through a program.
Definition 2.1 (PATHS). Given the CFG $G$ for a program $p$, a path $\pi$ is a sequence of consecutive edges of $G$ from the start node to the end node.

Each path $\pi$ defines a corresponding path condition $c(\pi)$ of $\pi$ : an input leads to the traversal of path $\pi$ if and only if it satisfies $c(\pi)$. The path $\pi$ is feasible if and only if there is an input that satisfies $c(\pi)$.

\subsection{Forms of program equivalence}

\subsubsection{Functional Equivalence}

A program $p$ is functionally equivalent to program $p^{\prime}$ if the external functional behaviour of the two programs is indistinguishable, so that for all inputs $i$, applying $p$ to $i$ gives the same result as applying $p^{\prime}$ to $i$. Functional equivalence requires identical nontermination behaviour: if $p$ fails to terminate on input $u$, then so does $p^{\prime}$. It does not require equivalence of non-functional behaviour. For example, $p$ and $p^{\prime}$ may take a different number of steps to produce the output. Crucially, functional equivalence is an extensional notion; it imposes no requirements on the internal implementation details of $p$ and $p^{\prime}$.

We can also define a restricted notion of functional equivalence, where we consider the equivalence of the behaviours for a subset of the possible inputs.

With program transformations, we may wish to preserve some aspects of the internal structure of the program. To this end, we require an intensional notion of equivalence.

\subsubsection{Path Equivalence}

One intensional aspect of a program that we may wish to preserve is the nature of the paths, or execution traces, of a program. There are various ways of defining path equivalence. We will state the strictest notion, which is defined in terms of a program's control flow graph.

Two programs $p, p^{\prime}$ are CFG-path equivalent if for every execution trace in the programs' control flow graphs, the same sequence of statements is executed under the same conditions. With CFG-path equivalence there is no attempt to consider which paths represent computations that can be realised: it includes paths that can never be executed because the path condition can never be met.

\subsection{Structured programs}

In the context of this paper, a structured program is one that does not exploit exit statements.

Definition 2.2 (Structured Program). $A$ structured program is one in which the only control flow that is permitted is that which is expressed by if-then-else-fi conditionals, single-exit while-do-od loops, and statement sequencing.

All other programs are considered to be unstructured. 
Definition 2.3 (Unstructured Program). An unstructured program is one that contains one or more exit statements.

Implicitly, this can be taken to include all programs containing other forms of arbitrary jump statements, such as goto. Such programs can be transformed into path equivalent programs containing exit statements and no goto statements, and with no additional variables [21].

Following from these definitions, a program is either structured or unstructured; for the purposes of this paper, there is no notion of one program being "more structured" than another.

\section{BRANCH COVERAGE}

This paper concentrates on one commonly used notion of coverage: branch coverage. A branch is an edge in the control flow graph, from a node $n$ where $n$ has more than one edge leaving it. Branches are thus associated with constructs such as if statements and loops. There is an additional branch from the start node: every path passes through this branch. Branch coverage may be defined in the following manner.

Definition 3.1 (Branch Coverage). A test input $t$ covers a branch $b$ of program $p$ if when $p$ is tested with $t$, the flow of control passes through $b$. A set $T$ of test inputs covers a branch $b$ of program $p$ if some $t \in T$ covers $b$.

Definition 3.2 (Proportion of Branches Covered). Given a set $T$ of test inputs and program $p$, the branch coverage of $p$ achieved by $T$, as a percentage, is:

$$
\frac{\text { Number of branches of } p \text { covered by } T}{\text { Number of feasible branches in } p} \times 100
$$

There has been a significant amount of work on the problem of automatically generating a set of test inputs that provides $100 \%$ branch coverage. While this problem is generally uncomputable, several approaches have proved to be effective in practice (see, for example, $[25,8,9,11,26,14,27])$.

Structural test criteria, such as branch coverage, base the requirements for testing on the structure of the code. Thus, there is a potential danger in transforming code to make it structured: if we transform an unstructured program $p$ to form a structured program $p^{\prime}$, a set of test inputs generated to cover the branches of $p^{\prime}$ does not need to cover the branches of $p$. Thus, we require a set of transformations such that:

1. The transformations will take unstructured code and return structured code.

2. If program $p$ is transformed into $p^{\prime}$ then any set of test inputs that gives $100 \%$ branch coverage for $p^{\prime}$ will also achieve $100 \%$ branch coverage for $p$.
It is requirement 2 above which makes this problem different from the traditional problem of restructuring unstructured programs.

We require a transformation system that converts unstructured code into structured code but that preserves something other than functional equivalence. Note that in principle it is not necessary to preserve functional equivalence. In order to illustrate this, consider the following simple program in which $S_{1}$ and $S_{2}$ contain no conditionals and thus no branches.

if $P$ then
$S_{1}$
else
$S_{2}$
fi

Since we are only concerned with preserving branchcoverage adequate sets of test inputs, it is legitimate to transform this into the following program since a set of test inputs covers all branches of one if and only if it covers all branches of the other.

\begin{tabular}{|l|}
\hline if $P$ then \\
$S_{2}$ \\
else \\
$S_{1}$ \\
fi
\end{tabular}

While the transformations that preserve branch coverage do not need to preserve the functionality of a program, in practice it is useful if they do. This is because transformations that do not preserve the functionality of the transformed section $S$ of a program $p$ must consider the context in which $S$ lies in $p$. For example, the transformation given above does not need to be valid when applied to a section of a program since it may alter the branches covered after the section is executed. For this reason, the transformation rules introduced in this paper preserve functional equivalence.

While it is helpful for our transformations to preserve functional equivalence, we do not wish to impose this restriction since this eliminates potentially useful transformations. Consider, for example, a statement $s$ of a program $p$ with the property that for every branch $b$ of $p, s$ does not affect the condition under which $b$ is executed. We can define a transformation that deletes $s$ from $p$. Then since this statement does not affect the conditions under which branches are executed, this transformation preserves the sets of branch-coverage adequate test inputs. Where we can eliminate a significant number of statements in this way, such transformations could allow us to produce a program with a shorter execution time. This would help when applying techniques such as Genetic Algorithms that can require us to execute our program many thousands of times. Thus there are potentially useful transformations that do not preserve functional equivalence. 
As seen in Subsection 2.3.1, the extensional notion of functional equivalence is inadequate for our purpose since it allows the branch structure (and even the statement structure) of the original program to be altered dramatically; functional equivalence makes no structural (syntactic) requirements on the transformation process. Unfortunately, as shown in Section 4, previous results from existing work on restructuring transformations show that multiple-level (and even single-level) exit statements cannot be removed under path equivalence. This is the motivation for the introduction of branch-coverage equivalence in Section 5.

\section{RELATED WORK}

Work related to this paper falls into two categories: previous work on transformation to address the problem of poor structure and previous work on program transformation to improve software testability. There is a large amount of prior work on the former, but comparatively little on the latter. This section first summarizes previous work on testability and transformation to improve testability before moving on to present an overview of related work on restructuring transformations.

Testability has been defined by Voas [28] in terms of the Propagation, Infection and Execution (PIE) framework. The PIE method, a mutation testing-based approach, measures testability in terms of the likelihood that an infection (a fault) is executed and subsequently propagated in an observable way. Voas was concerned with testability measurement.

Harman et al. [23] introduce testability transformation; the transformation of programs with the goal of improving the ability to test the programs. Testability transformation focuses upon ameliorating problems for automated test-data generation techniques. The novel aspects of testability-transformation theory are discussed in [23], where it is noted that new forms of equivalence are required by testability transformation. In this paper, we introduce an example of a testability transformation that requires such a new form of equivalence and a new proof obligation.

There is a large body of existing literature on the goto removal problem (for example see [29, 30, 31, $32,33,34,21,35])$. Much of this work considers the problem to be one of removing goto statements, replacing them with programs which retain the exit (or break) statements though they have no goto statements.

Some authors consider such exit statements to be 'structured' forms of goto $[17,36]$. For the purpose of test-data generation, the aim is to replace all unstructuredness (including exit statements) to create single-entry, single-exit constructs. Previous work which retains exit statements is not, therefore, directly useful. However, previous work on multiple-level exits is useful because it establishes the expressiveness of these constructs, in particular it shows that:

- Multiple-level exit statements capture the full expressive power of arbitrary unstructuredness (and that single-level exit statements do not);

- goto statements cannot always be removed while preserving path equivalence.

The first of these motivates our choice of multiplelevel exit statements as the paradigm of unstructuredness to be considered, while the second motivates the need for additional transformations which, while not path-equivalence preserving, nonetheless, preserve aspects of the structure of a program.

\subsection{Restructuring transformations}

Ramshaw [21] presents an important result concerning multiple-level exit statements:

THEOREM 4.1 (RAMSHAW, 1985). Under path equivalence an arbitrary goto program can be transformed into one with no goto statements, but which may contain multiple-level exit statements.

This result shows that multiple-level exit statements are powerful enough to capture the expressiveness of goto statements; no new variables need be introduced and the program's structure (the set of paths it traverses) is preserved. Clearly, path preservation implies branch preservation. It is upon this result that we rest our claim to consider the full generality of the unstructuredness problem for branch-coverage in the present paper.

Previous work has also shown that multiple-level exit statements are more powerful than single-level exit statements. This result is due ${ }^{5}$ to Peterson [34].

Theorem 4.2 (Peterson, 1973). Under path equivalence, it is not always possible to transform an arbitrary goto program to one with no goto statements, but which may contain single-level exit statements.

Knuth, Floyd and Hopcroft show ${ }^{6}$ that it is not possible to remove goto statements from arbitrary unstructured programs while preserving path equivalence.

Theorem 4.3 (KnUth, Floyd And Hopcroft, 1971). There are programs, the gotos of which cannot be eliminated under path equivalence.

Knuth and Floyd use 'regular expression semantics' for flowcharts. The regular expression captures the possible paths through the flowchart, thereby capturing path equivalence. They then define a regular expression class, $\mathcal{R}$ which describes paths through programs in a

\footnotetext{
${ }^{5}$ Ramshaw [21] claims that this result is due to Kosaraju [33] in 1974. However, it appears that the same result was shown by Peterson in 1973.

${ }^{6}$ In [36], the proof of this result is attributed to J. Hopcroft, although Hopcroft is not an author of the paper.
} 


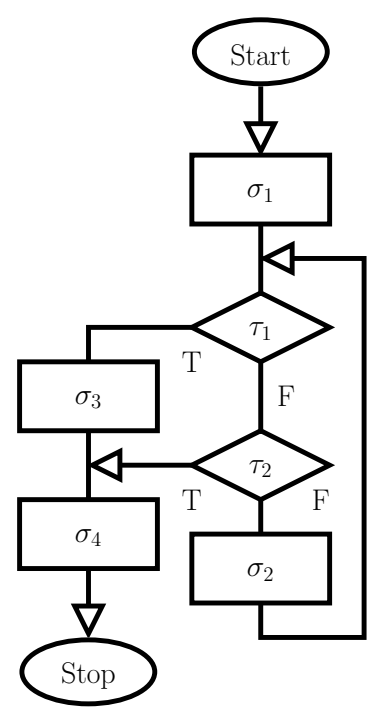

FIGURE 3. Knuth, Floyd and Hopcroft's Example

language which is essentially that used in the present paper, but with repeat loops. The addition of repeat loops is not significant. Knuth and Floyd show that the following regular expression cannot be converted into an equivalent one in $\mathcal{R}$ :

$$
\sigma_{1}\left(\tau_{1 \mathrm{~F}} \tau_{2 \mathrm{~F}} \sigma_{2}\right)^{*}\left(\tau_{1 \mathrm{~T}} \sigma_{3} \mid \tau_{1 \mathrm{~F}} \tau_{2 \mathrm{~T}}\right) \sigma_{4}
$$

The flow chart corresponding to this regular expression is depicted in Figure 3. As can readily be seen, this flowchart is not in some way 'pathological'. The problem goto is simply a jump out of a while loop that targets a point a little further down (lexically) from the end of the while loop.

Since we know that any goto program may be converted into a path equivalent program in which the only unstructuredness is due to exit statements, we have the following result.

TheOrem 4.4. Programs exist whose only unstructuredness is due to exit statements, that are not path equivalent to any structured program.

This result provides the motivation for the definition of a new notion of equivalence and branch-coverage preserving transformations. The transformations introduced do not preserve path equivalence, while they do preserve branch-coverage adequate sets of test inputs.

\section{BRANCH-PRESERVING TRANSFORM- ATIONS}

This section defines what it means for a transformation to preserve branch coverage. It will thus state a requirement for the transformation system, based on a new notion of equivalence, that will be developed in Section 6. First, we will introduce some notation.
Definition 5.1 (Program Branches). Given a program $p, B(p)$ will denote the branches of $p$.

Definition 5.2 (Branch Coverage Condition). For each branch $b \in B(p)$ there is an associated condition $c(b, p)$ on the input to $p$ such that: test input $t$ leads to $b$ being covered if and only if $c(b, p)(t)$ is true. The expression $c(b, p)(t)$ will also be written $c(b, p, t)$.

We wish to transform a program $p$ into a program $p^{\prime}$ that has the property that if we generate a set $T$ of test inputs that provides $100 \%$ branch coverage for $p^{\prime}$ then $T$ satisfies $100 \%$ branch coverage for $p$. This relation between $p^{\prime}$ and $p$ is captured by the following definition.

Definition 5.3 (Branch Coverage SubsumpTION). Program $p^{\prime}$ branch-coverage subsumes $p$ if and only if for every set $T$ of test inputs, if T provides $100 \%$ branch coverage for $p^{\prime}$ then $T$ provides $100 \%$ branch coverage for $p$.

Naturally, we only need to consider feasible branches.

Definition 5.4 (Feasible Branches). Branch $b$ of program $p$ is feasible if and only if $\exists t . c(b, p, t)$. The set of feasible branches of $p$ will be denoted $\bar{B}(p)$.

Proposition 5.1. Program $p^{\prime}$ branch-coverage subsumes program $p$ if and only if the following holds:

$$
\forall b \in \bar{B}(p) . \exists b^{\prime} \in \bar{B}\left(p^{\prime}\right) . c\left(b^{\prime}, p^{\prime}, t\right) \Rightarrow c(b, p, t)
$$

Proof. Case 1: $\Rightarrow$

Suppose $p^{\prime}$ branch-coverage subsumes program $p$ and $b \in \bar{B}(p)$. Proof by contradiction: suppose that for all $b^{\prime} \in \bar{B}\left(p^{\prime}\right)$ we have that $c\left(b^{\prime}, p^{\prime}, t\right) \nRightarrow c(b, p, t)$.

Now choose a set of test inputs in the following way. For every $b^{\prime} \in \bar{B}\left(p^{\prime}\right)$ choose a test input $t_{b^{\prime}}$ such that $c\left(b^{\prime}, p^{\prime}, t_{b^{\prime}}\right) \wedge \neg c\left(b, p, t_{b^{\prime}}\right)$. Since $c\left(b^{\prime}, p^{\prime}, t\right) \nRightarrow c(b, p, t)$, there must be some such $t_{b^{\prime}}$. Then the resultant set $T$ of test inputs provides $100 \%$ branch coverage for $p^{\prime}$ but does not cover the feasible branch $b$ of $p$. This contradicts the assumption that $p^{\prime}$ branch-coverage subsumes program $p$ as required.

Case $2: \Leftarrow$

Suppose that for all $b \in \bar{B}(p)$ there exists $b^{\prime} \in \bar{B}\left(p^{\prime}\right)$ such that $c\left(b^{\prime}, p^{\prime}, t\right) \Rightarrow c(b, p, t)$. Proof by contradiction: suppose that $p^{\prime}$ does not branch-coverage subsume program $p$. Then there exists a set $T$ of test inputs that provides $100 \%$ branch coverage for $p^{\prime}$ but does not provide $100 \%$ branch coverage for $p$. Suppose that $T$ does not cover the feasible branch $b$ of $p$. It is now sufficient to note that $\exists b^{\prime} \in \bar{B}\left(p^{\prime}\right) . c\left(b^{\prime}, p^{\prime}, t\right) \Rightarrow c(b, p, t)$ and, since $T$ covers all branches of $p^{\prime}$, there is some $t_{b^{\prime}} \in T$ such that $c\left(b^{\prime}, p^{\prime}, t_{b^{\prime}}\right)$. Thus $c\left(b, p, t_{b^{\prime}}\right)$ and so $T$ must cover $b$, providing a contradiction as required.

If $p^{\prime}$ branch-coverage subsumes $p$ then, if we want a set of test inputs to cover all the feasible branches of $p$ it is sufficient to produce a set of test inputs that covers all feasible branches of $p^{\prime}$. However, in producing a set 
of test inputs for $p^{\prime}$ we may be achieving more than is required. This observation motivates the following desirable property.

Definition 5.5 (Branch Coverage EquivaLENCE). Program $p^{\prime}$ is branch-coverage equivalent to $p$ if and only if for every set $T$ of test inputs, $T$ provides $100 \%$ branch coverage for $p^{\prime}$ if and only if $T$ provides $100 \%$ branch coverage for $p$.

\section{A TRANSFORMATION ALGORITHM}

This section will define a transformation algorithm that takes a program $p$ that contains one or more exit statements and transforms this to a branch-coverage equivalent program $p^{\prime}$ that does not contain any exit statements. The exit statements may be multiplelevel, but each loop may only have a single exit statement which exits the loop. Future work will consider more general transformation algorithms and transformation rules that take a program containing loops with more than one exit statement and return a branch-coverage equivalent program in which each loop contains at most one exit statement. Consider, for example, the following program.

\begin{tabular}{|l|}
\hline while $P$ do \\
S \\
if $P^{\prime}$ then \\
$S_{1} ;$ \\
exit $n ;$ \\
$S_{2} ;$ \\
else \\
$S_{3} ;$ \\
exit $n ;$ \\
$S_{4} ;$ \\
fi \\
$S_{5} ;$ \\
od \\
\hline
\end{tabular}

This is branch-coverage equivalent to the following program that contains only one exit statement in its loop.

\begin{tabular}{|l|} 
while $P$ do \\
$S$ \\
if $P^{\prime}$ then \\
$S_{1} ;$ \\
else \\
$S_{3} ;$ \\
fi \\
exit $n ;$ \\
$S_{5} ;$ \\
od
\end{tabular}

It will be assumed that all expressions in each predicate of $p$ (of either an if statement or a while loop) contained in a loop that has an exit statement are side-effect free — they cannot alter the value of any program variable. Where a program $p$ contains predicates with side-effects, it may be transformed to form a program $p^{\prime}$ that does not contain such side-effects [37, 38]. The problem of producing a complete set of branch-coverage equivalence preserving transformations that remove side-effects from predicates will form a significant element of future work.

This section is structured as follows. Subsection 6.1 gives a set of transformation rules which are used in Subsection 6.2 to define a transformation algorithm. Subsection 6.3 provides a proof of correctness and Subsection 6.4 proves that the algorithm has low-order polynomial time complexity.

\subsection{Transformation rules}

In order to simplify the exposition, the only control structures considered in this paper are while-do-od loops and if-then-else-fi statements. Naturally, if-then-fi structures can be dealt with by applying the following branch-coverage equivalence preserving transformation.

Rule 1. The following may be applied to an if-then-fi statement.

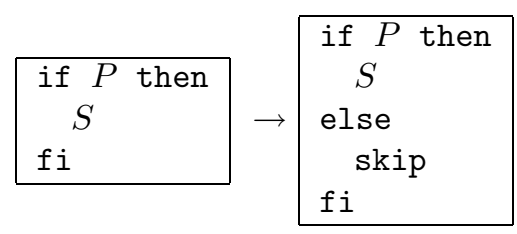

We argue that other standard control constructs may be dealt with using rules similar to those described here.

At times we will want to talk about a subprogram of a program $p$. This will be defined in the following way.

Definition 6.1 (SubProgram). The concept of one program being a subprogram of another is defined by the following rules.

1. $S$ is a subprogram of $S$.

2. Given any program $p$, skip is a subprogram of $p$.

3. $S$ is a subprogram of $S ; S^{\prime}$.

4. $S$ is a subprogram of $S^{\prime} ; S$.

5. $S_{1}$ is a subprogram of if $P$ then $S_{1}$ else $S_{2}$ $f i$.

6. $S_{2}$ is a subprogram of if $P$ then $S_{1}$ else $S_{2}$ $f i$.

7. $S$ is a subprogram of while $P$ do $S$ od.

8. If $p^{\prime}$ is a subprogram of $p$ and $p^{\prime \prime}$ is a subprogram of $p^{\prime}$ then $p^{\prime \prime}$ is a subprogram of $p$.

We say that $S_{1}$ is contained in $S_{2}$ if $S_{1}$ is a subprogram of $S_{2}$. Each exit statement is contained within the body of a loop. At points, when considering a statement $s$ we will want to talk about the body of the innermost loop that contains $s$.

Definition 6.2 (InNermost Loop Body). Given exit statement $s$ in program $p$, $\operatorname{subprog}(s, p)$ is the 
maximum subprogram, $S$, of $p$ such that $s$ is contained in $S$ and $s$ is not contained within a loop in $S$.

Where a statement $s$ lies within the body of the loop while $P$ do $S$ od, and is not contained in a loop within $S$, it is possible to define the depth, $\operatorname{depth}(s, S)$ of $s$ in this loop. The term $\operatorname{depth}(s, S)$ will denote how far $s$ is nested, inside if statements, within $S$.

Definition 6.3 (Depth). The function depth is defined by the following rules.

$$
\begin{aligned}
& \operatorname{depth}(s, s) \\
& \operatorname{depth}\left(s, S ; S^{\prime}\right) \\
& \operatorname{depth}\left(\begin{array}{c}
\text { if } P \\
\text { then } S \\
\text { else } S^{\prime} \\
\text { fi }
\end{array}\right)=\left\{\begin{array}{c}
\operatorname{depth}(s, S) \\
\text { if } s \text { is in } S \\
\operatorname{depth}\left(s, S^{\prime}\right) \\
\text { otherwise } \\
1+\operatorname{depth}(s, S) \\
\text { if } \sin \text { in } S \\
1+\operatorname{depth}\left(s, S^{\prime}\right) \\
\text { otherwise }
\end{array}\right.
\end{aligned}
$$

The precondition for depth $(s, S)$ is that $s$ is an exit statement contained within a loop-free section of $S$.

The above definition assumes that exit statements are uniquely identified: where there are two syntactically equivalent exit statements, we label these in order to distinguish between them.

Definition 6.4 (DEPTH OF AN EXIT). Given exit statement $s$ in program $p$, the depth of $s$ in $p$ is $\operatorname{depth}(s, S)$, where $S=\operatorname{subprog}(s, p)$.

We now introduce transformations rules that will be used in order to eliminate (possibly multiple-level) exit statements while preserving branch-coverage equivalence. In order to do this we consider the following cases:

1. an exit statement of depth 0 ;

2. an exit statement of depth 1 ;

3. an exit statement of depth greater than 1 .

We give transformation steps for each of these cases and assume that an exit statement $s$ is represented as exit $n$, where $n$ denotes the number of levels over which the exit operates. Essentially, the algorithm takes exit statements and repeatedly transforms the program in order to reduce their level. Once the level of an exit statement has been reduced to 0 it is replaced by skip.

A single-level exit will be represented by exit 1 . It will transpire that the transformation rules may reduce a single-level exit to the statement exit 0 . We thus introduce the following transformation that cleans up any such terms generated in the transformation process.

Rule 2.

$$
\text { exit } 0 \rightarrow \text { skip }
$$

The following are immediate.
Proposition 6.1. If $p$ may be transformed into $p^{\prime}$ using an application of Rule 2 then $p^{\prime}$ is branch-coverage equivalent to $p$.

Proposition 6.2. If $p$ may be transformed into $p^{\prime}$ using an application of Rule 2 then $p^{\prime}$ and $p$ are functionally equivalent.

While functional equivalence is not required for branch-coverage equivalence, it is useful to have functional-equivalence preserving transformations since these cannot affect the behaviour of the program on any code executed after the transformed fragment, making the application of the transformations context independent.

Rule 3. The following rule may be applied to an exit statement of depth 0 .

$$
\begin{array}{|l|l|}
\hline \text { while } P \text { do } P \text { then } \\
S ; \\
\text { exit } n ; \\
S^{\prime} \\
\text { od }
\end{array} \rightarrow \begin{array}{|}
\text { exit } n-1 \\
\text { else } \\
\text { skip } \\
\text { fi }
\end{array}
$$

This has the precondition that $n>0$.

Proposition 6.3. If $p$ may be transformed into $p^{\prime}$ using an application of Rule 3 then $p^{\prime}$ is branch-coverage equivalent to $p$.

Proof. Suppose that the rule is applied to a subprogram $X_{1}$ of $p$ of the form

$$
\begin{aligned}
& \text { while } P \text { do } \\
& \quad S ; \\
& \quad \text { exit } n ; \\
& S^{\prime} \\
& \text { od }
\end{aligned}
$$

to create a subprogram $X_{2}$ of $p^{\prime}$ of the form

if $P$ then
$\quad S ;$
exit $n-1$
else
$\quad$ skip
fi

Clearly paths in $p$ that do not pass through $X_{1}$ are not affected by this transformation. Consider a feasible path $\pi$ through $p$ that passes through $X_{1}$. Here it is important to observe that the loop may iterate at most once.

Each time $X_{1}$ is met on $\pi$, there are two cases to consider:

1. $P$ is true in the state before $X_{1}$ is executed. In this case the path passes through $S$ and then executes exit $n$.

2. $P$ is false in the state before $X_{1}$ is executed. The path $\pi$ then exits this section of the code. 
Now consider $X_{2}$. Each time $X_{2}$ is met in a path, there are two cases to consider:

1. $P$ is true in the state before $X_{2}$ is executed. In this case the path passes through $S$ and then executes exit $n-1$.

2. $P$ is false in the state before $X_{2}$ is executed. The path then exits this section of the code.

The statement exit $n$, in $X_{1}$, is equivalent to the statement exit $n-1$ in $X_{2}$. Thus, $X_{1}$ and $X_{2}$ are functionally equivalent. In addition, the branches in $X_{1}$ and $X_{2}$ are followed under the same conditions. The result thus follows.

The following may be proved in a similar manner.

Proposition 6.4. If $p$ may be transformed into $p^{\prime}$ using an application of Rule 3 then $p^{\prime}$ and $p$ are functionally equivalent.

RulE 4. The following may be applied to an exit statement of depth 1 within the then part of an if statement.

\begin{tabular}{|c|c|}
\hline while $P$ do \\
S; \\
if $P^{\prime}$ then \\
$S_{1} ;$ \\
exit $n ;$ \\
$S_{2}$ \\
else \\
$S_{3}$ \\
fi \\
$S_{4}$ \\
od
\end{tabular}$\quad$\begin{tabular}{|c} 
while $\left(P \wedge \neg P^{\prime \prime}\right)$ do \\
$S ;$ \\
$S_{3} ;$ \\
od; $P$ then \\
if $S ;$ \\
$S_{1} ;$ \\
exit $n-1$ \\
fi
\end{tabular}

Here $P^{\prime \prime}$ denotes the result of evaluating $P^{\prime}$ after executing $S . P^{\prime \prime}$ is described in more detail below. This rule has the precondition that $n>0$.

Rule 4 is illustrated by Figure 4 .

The transformation which produces $P^{\prime \prime}$ from $S$ and $P^{\prime}$ consists of making $P^{\prime \prime}$ a call to a new function $\phi$. The local variables of $\phi$ are the variables of $S$ which are defined before they are used [39]. The formal (value) parameters of $\phi$ are the variables of $S$ which are not defined before use (that is those either used before they are defined or those simply used and not defined at all).

The function $\phi$ is a predicate; it returns a Boolean result. The body of $\phi$ consists of the statement block $S$, followed by a return statement. The return statement simply returns the result of evaluating the expression $P^{\prime}$.

Observe that $P^{\prime}$ is guaranteed to have the same meaning in the context of the return statement from $\phi$ as it does from the original point in the program at which it occurs. Any variable (mentioned in $P^{\prime}$ ) which is defined before it is used can be replaced by a local variable, since its value is defined before use this will not lose any previous value. Any variable (mentioned in
$P^{\prime}$ ) which is used before it is defined, will be passed (by value) to $\phi$, thereby creating a local copy. Any variable simply used and not defined will be passed as a formal parameter. Clearly a variable must be either defined before it is used or not and so all variables mentioned in $P^{\prime}$ will be available (with their correct value) at the point of the return statement.

Also, observe that the call to the function $\phi$ has no side-effects, since all variables which are defined by the copy of $S$ in the body of $\phi$ are either local or are formal value parameters. These transformations assume that the statement $S$ performs no input/output, or any other implicit [40] state-update and that the expression $P^{\prime}$ is side-effect free.

Proposition 6.5. If $p$ may be transformed into $p^{\prime}$ using an application of Rule 4 then $p^{\prime}$ is branch-coverage equivalent to $p$.

Proof. Suppose that the rule is applied to a subprogram $X_{1}$ of $p$, of the form

\begin{tabular}{|l|}
\hline while $P$ do \\
S; \\
if $P^{\prime}$ then \\
$S_{1} ;$ \\
exit $n ;$ \\
$S_{2}$ \\
else \\
$S_{3}$ \\
fi \\
$S_{4}$ \\
od
\end{tabular}

to create a subprogram $X_{2}$ of $p^{\prime}$ of the form

\begin{tabular}{|l|}
\hline while $\left(P \wedge \neg P^{\prime \prime}\right)$ do \\
$\quad S ;$ \\
$S_{3} ;$ \\
$S_{4}$ \\
od; \\
if $P$ then \\
$\quad S ;$ \\
$\quad S_{1} ;$ \\
exit $n-1$ \\
fi
\end{tabular}

Clearly paths in $p$ that do not pass through $X_{1}$ are not affected by this transformation. Consider a path $\pi$ of $p$ that passes through $X_{1}$. Consider a point at which the path $\pi$ passes through $X_{1}$.

Observe that the conditions for leaving the loops in $X_{1}$ and $X_{2}$ are equivalent.

There are now two cases to consider for $X_{1}$ :

1. The loop terminates due to $P$ being false, or becoming false. Thus, the program passes through $S ; S_{3} ; S_{4}$ zero or more times until $P$ is false.

2. The loop terminates due to $P^{\prime}$ becoming true. Thus, the program repeatedly passes through $S ; S_{3} ; S_{4}$, then $S$ followed by $P^{\prime}$ being true. The 


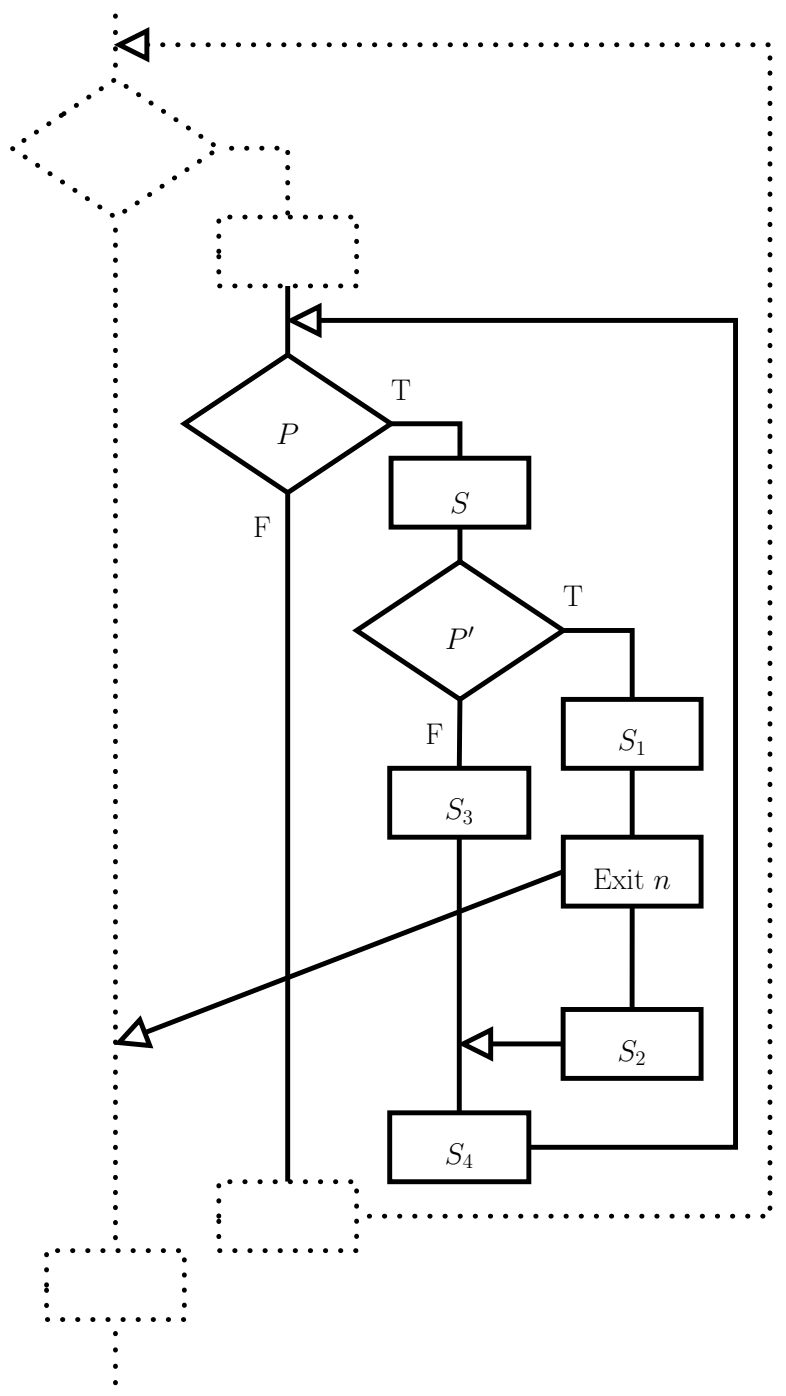

Before

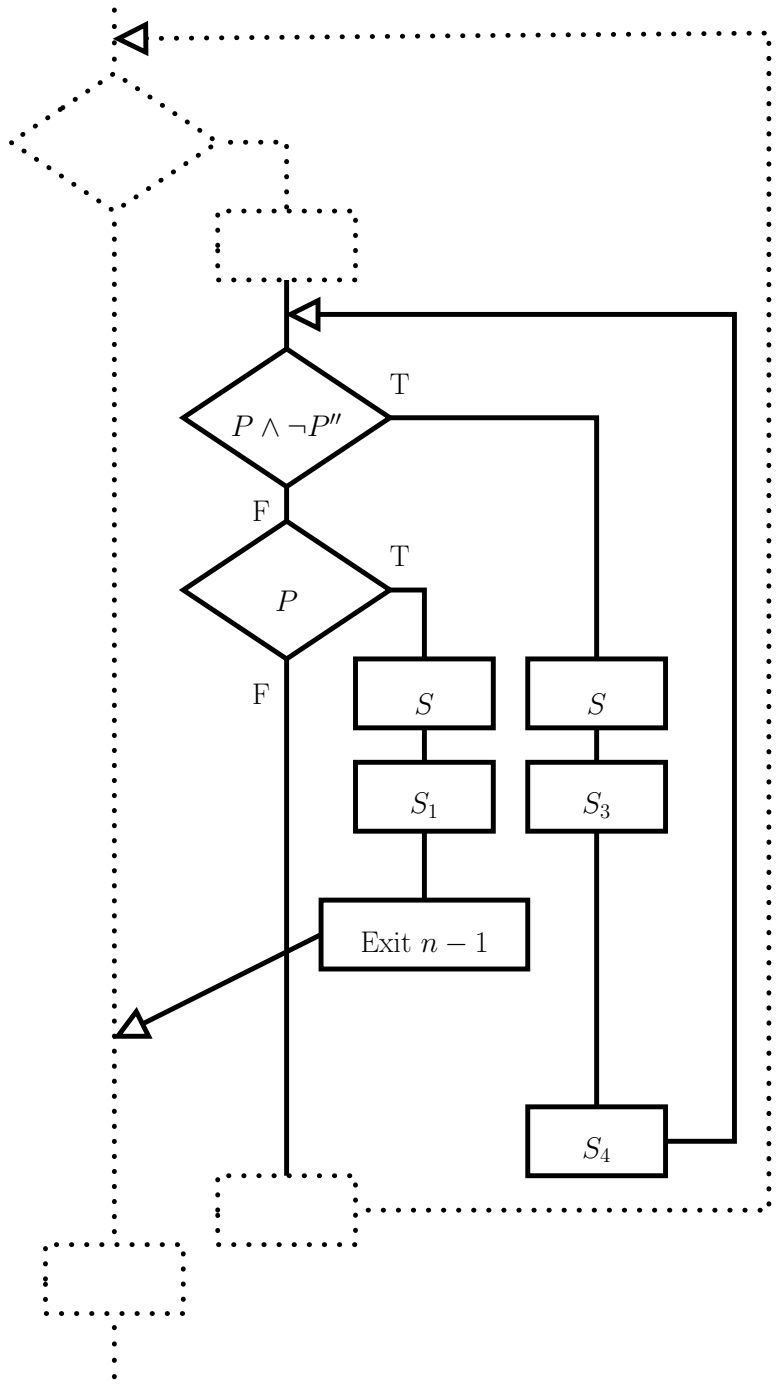

After

FIGURE 4. Illustration of Rule 4, showing the reduction in depth of the exit statement

program then passes through $S_{1}$ before meeting exit $n$.

Now consider $X_{2}$. Again there are two cases.

1. The loop is left due to $P$ being false, or becoming false. Thus, the program passes through $S ; S_{3} ; S_{4}$ zero or more times until $P$ is false.

2. The loop is left due to $\neg P^{\prime \prime}$ becoming false. In this case, upon leaving the loop $P$ must be true and thus this is followed by $S ; S_{1}$; exit $n-1$. Thus, the program repeatedly passes through $S ; S_{3} ; S_{4}$, then $S$ followed by $P^{\prime}$ being true. The program then passes through $S_{1}$ and meets exit $n-1$.

The branches contained in $P^{\prime \prime}$ are equivalent to those in $S$ and are executed under the same condition as $S$ in $X_{2}$. Thus we may ignore the branches in $P^{\prime \prime}$ when determining whether $100 \%$ branch coverage has been achieved for $p^{\prime}$.

The result now follows by observing that the statement exit $n$ in $X_{1}$ is equivalent to the statement exit $n-1$ in $X_{2}$ and the equivalence of the cases for $X_{1}$ and $X_{2}$; these correspond to branches.

A similar argument may be used to prove the following.

Proposition 6.6. If $p$ may be transformed into $p^{\prime}$ using an application of Rule 4 then $p^{\prime}$ is functionally equivalent to $p$.

The following is equivalent to Rule 4, except with the exit statement in the else part of the if statement.

Rule 5. The following may be applied to an exit statement of depth 1 within the else part of an if statement. 


\begin{tabular}{|c|}
\hline while $P$ do \\
$S ;$ \\
if $P^{\prime}$ then \\
$S_{1} ;$ \\
else \\
$S_{2} ;$ \\
exit $n ;$ \\
$S_{3}$ \\
fi \\
$S_{4}$ \\
od
\end{tabular}$\quad \rightarrow$\begin{tabular}{|c|}
\hline while $\left(P \wedge P^{\prime \prime}\right)$ do \\
$S ;$ \\
$S_{1} ;$ \\
$S_{4}$ \\
od; $P$ then \\
if $S ;$ \\
$S_{2} ;$ \\
exit $n-1$ \\
fi
\end{tabular}

Here $P^{\prime \prime}$ denotes the result of evaluating $P^{\prime}$ after executing $S$. This rule has the precondition that $n>0$.

The proofs of the following are similar to those of Propositions 6.5 and 6.6 .

Proposition 6.7. If $p$ may be transformed into $p^{\prime}$ using an application of Rule 5 then $p^{\prime}$ is branch-coverage equivalent to $p$.

Proposition 6.8. If $p$ may be transformed into $p^{\prime}$ using an application of Rule 5 then $p^{\prime}$ is functionally equivalent to $p$.

Where an exit statement $s$ has depth greater than 1 we may use Rule 6 , below, in order to reduce its depth. Thus, repeated application of Rule 6 may be used to reduce the depth of $s$ to 1 whereupon Rule 4 or Rule 5 may be applied.

Rule 6 . The following may be applied to an exit statement of depth greater than 1

\begin{tabular}{|c|c|}
\hline if $P$ then \\
$S_{1} ;$ \\
if $P^{\prime}$ then \\
$T_{1} ;$ \\
exit $n ;$ \\
$T_{2}$ \\
else \\
$T_{3} ;$ \\
fi \\
$S_{2}$ \\
else \\
$S_{3}$ \\
fi \\
else $\left(P \wedge P^{\prime \prime}\right)$ then \\
$T_{1} ;$ \\
if $P$ then \\
$S_{1} ;$ \\
$T_{3} ;$ \\
$S_{2}$ \\
else \\
fi $S_{3}$ \\
fi \\
\hline
\end{tabular}

$P^{\prime \prime}$ is the result of evaluating $P^{\prime}$ after executing $S_{1}$. This rule has the precondition that $n>0$.

Rule 6 is illustrated by Figure 5 .

Proposition 6.9. If $p$ may be transformed into $p^{\prime}$ using an application of Rule 6 then $p^{\prime}$ is branch-coverage equivalent to $p$.

Proof. Suppose that the rule is applied to a subprogram $X_{1}$ of $p$, of the form

\begin{tabular}{|l|}
\hline if $P$ then \\
$S_{1} ;$ \\
if $P^{\prime}$ then \\
$T_{1} ;$ \\
exit $n ;$ \\
$T_{2}$ \\
else \\
$T_{3}$ \\
fi \\
$S_{2}$ \\
else \\
$S_{3}$ \\
fi
\end{tabular}

to create a subprogram $X_{2}$ of $p^{\prime}$ of the form

if $\left(P \wedge P^{\prime \prime}\right)$ then
$S_{1} ;$
$T_{1} ;$
exit $n$
else
if $P$ then
$\quad S_{1} ;$
$T_{3} ;$
$S_{2}$
else
$S_{3}$
fi
fi

Paths in $p$ that do not pass through $X_{1}$ are not affected by this transformation. Consider a path $\pi$ of $p$ that passes through $X_{1}$. Consider a point at which $\pi$ passes through $X_{1}$. There are three cases.

1. $P$ is false and $S_{3}$ is executed.

2. $P$ is initially true and $P^{\prime}$ is true when evaluated after $S_{1}$. Here the program passes through $S_{1} ; T_{1}$; and then exit $n$.

3. $P$ is initially true and $P^{\prime}$ is false when evaluated after $S_{1}$. Here the program passes through $S_{1} ; T_{3} ; S_{2}$.

Now consider $X_{2}$. Again there are three cases.

1. $P \wedge P^{\prime \prime}$ is initially false and $P$ is initially false. Here $S_{3}$ is executed.

2. $P \wedge P^{\prime \prime}$ is initially true. Here the program passes through $S_{1} ; T_{1}$; and then exit $n$.

3. $P \wedge P^{\prime \prime}$ is initially false and $P$ is initially true. Here the program passes through $S_{1} ; T_{3} ; S_{2}$.

The branches in $P^{\prime \prime}$ are equivalent to those in $S_{1}$ in $X_{2}$ and are executed under the same conditions $(P$ being true).

The result follows by observing that the three cases for $X_{2}$ are equivalent to the three cases for $X_{1}$, and that in each case the conditions defined by the cases correspond to the branches. 


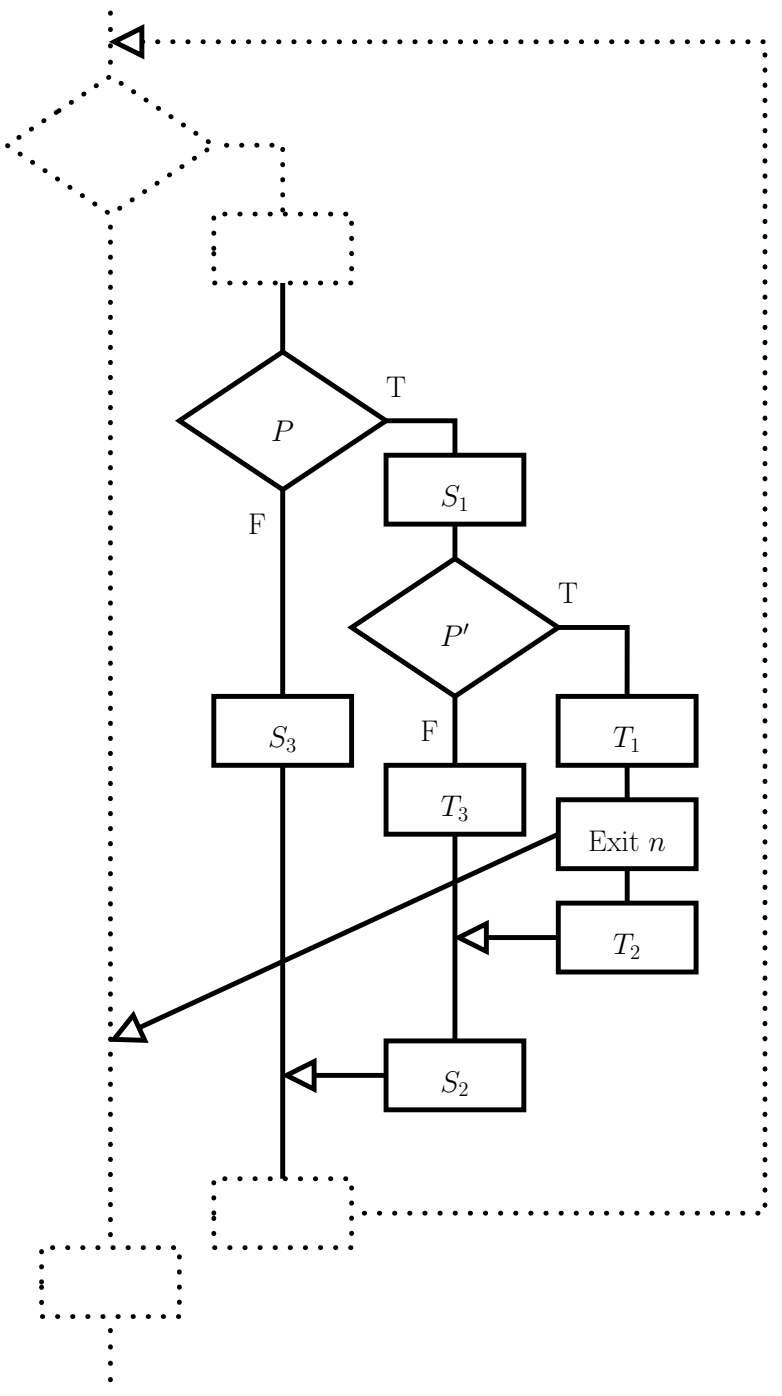

Before

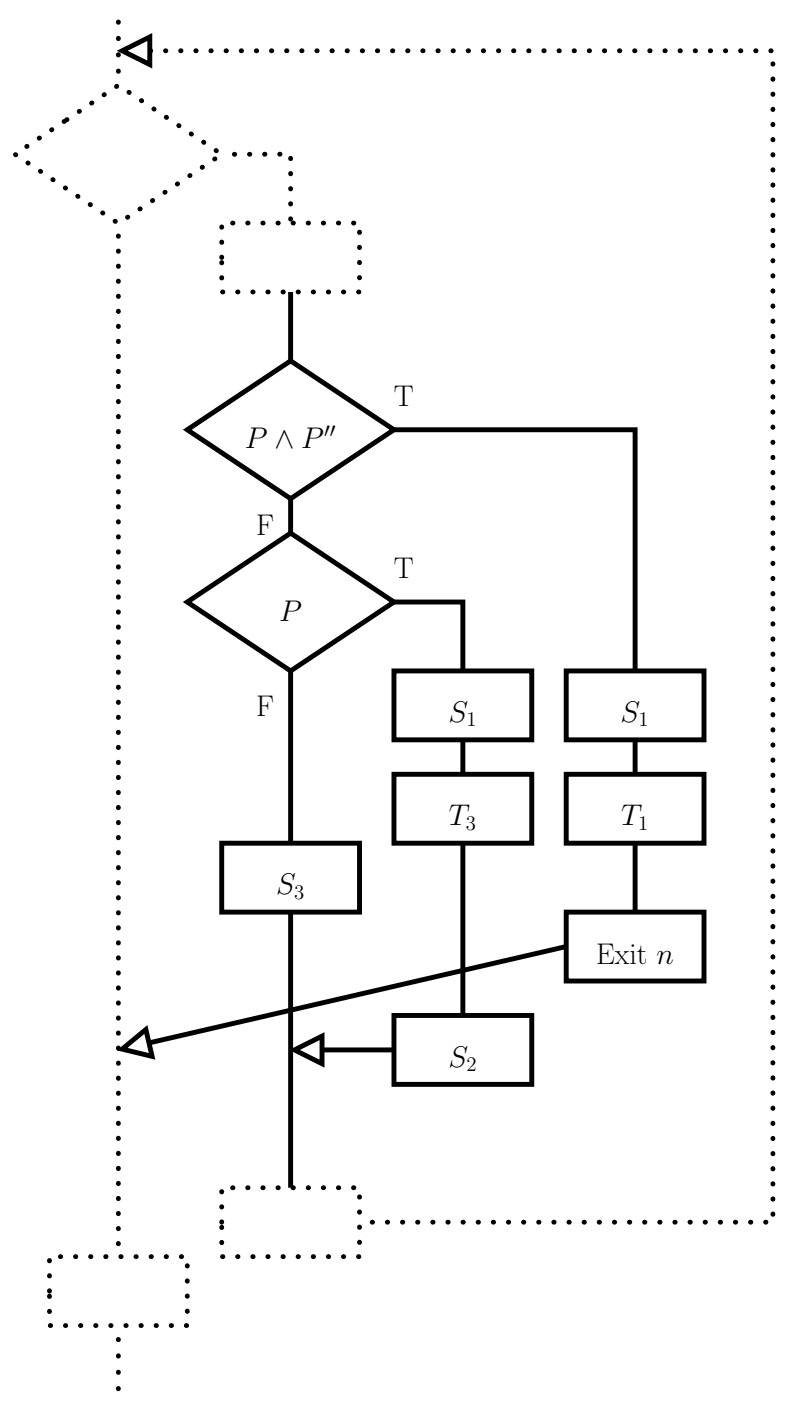

After

FIGURE 5. Illustration of Rule 6

Proposition 6.10. If $p$ may be transformed into $p^{\prime}$ using an application of Rule 6 then $p^{\prime}$ is functionally equivalent to $p$.

The transformation rule only deals with exit statements in the then case of an if statement. We thus get three additional rules. These will now be stated.

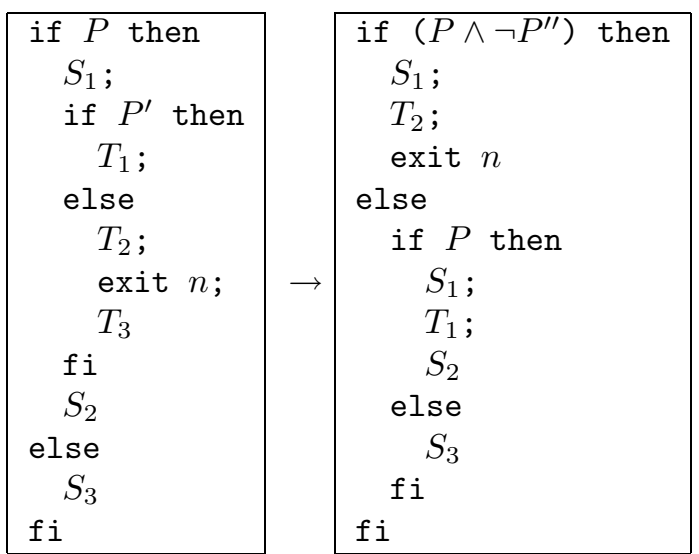

$P^{\prime \prime}$ is the result of evaluating $P^{\prime}$ after executing $S_{1}$. This rule has the precondition that $n>0$.

Rule 8. The following may be applied to an exit statement of depth greater than 1 .
Rule 7 . The following may be applied to an exit statement of depth greater than 1. 


\begin{tabular}{|c|c|}
\hline if $P$ then & if $\left(\neg P \wedge P^{\prime \prime}\right)$ then \\
$S_{1}$ & $S_{2} ;$ \\
else & $T_{1} ;$ \\
$S_{2} ;$ & exit $n$ \\
if $P^{\prime}$ then \\
$T_{1} ;$ \\
exit $n ;$ \\
$T_{2}$ \\
else \\
$T_{3}$ \\
fi \\
$S_{3}$ \\
fi
\end{tabular}$\quad \rightarrow \mid \begin{gathered}S_{2} ; \\
T_{3} ; \\
S_{3} \\
\text { else } \\
S_{1} \\
\mathrm{fi} \\
\mathrm{fi}\end{gathered}$

$P^{\prime \prime}$ is the result of evaluating $P^{\prime}$ after executing $S_{2}$. This rule has the precondition that $n>0$.

RulE 9. The following may be applied to an exit statement of depth greater than 1.

\begin{tabular}{|c|}
\hline if $P$ then \\
$S_{1}$ \\
else \\
$S_{2} ;$ \\
if $P^{\prime}$ then \\
$T_{1}$ \\
else \\
$T_{2} ;$ \\
exit $n ;$ \\
$T_{3}$ \\
fi \\
$S_{3}$ \\
fi
\end{tabular} \mid \begin{tabular}{c|} 
if $\left(\neg P \wedge \neg P^{\prime \prime}\right)$ then \\
$S_{2} ;$ \\
exit $n$ \\
else \\
if $\neg P$ then \\
$S_{2} ;$ \\
$T_{1} ;$ \\
$S_{3}$ \\
else \\
$S_{1}$ \\
fi \\
fi
\end{tabular}

$P^{\prime \prime}$ is the result of evaluating $P^{\prime}$ after executing $S_{2}$. This rule has the precondition that $n>0$.

The proofs of the following are similar to those of Propositions 6.9 and 6.10 .

Proposition 6.11. If $p$ may be transformed into $p^{\prime}$ using an application of Rule 7, Rule 8, or Rule 9 then $p^{\prime}$ is branch-coverage equivalent to $p$.

Proposition 6.12. If $p$ may be transformed into $p^{\prime}$ using an application of Rule 7, Rule 8, or Rule 9 then $p^{\prime}$ is functionally equivalent to $p$.

\subsection{Transformation Algorithm}

This section will describe a transformation algorithm based on the rules defined in Subsection 6.1. The algorithm is structured in order to guarantee that each rule's precondition holds when it is applied. Subsection 6.3 proves that this algorithm is correct and Subsection 6.4 explores its algorithmic complexity.

The function $t d e p t h$, which gives the nesting level of a statement in a program, will be used in the algorithm in order to ensure that the most deeply nested exit statements are transformed out first.

Definition 6.5 (TDEPTH). The function tdepth is defined by the following rules.

$$
\begin{aligned}
& \operatorname{tdepth}(s, s) \quad=0 \\
& \operatorname{tdepth}\left(s, S ; S^{\prime}\right) \\
& =\left\{\begin{array}{c}
t \text { depth }(s, S) \\
\text { if } s \text { is in } S \\
t \text { depth }\left(s, S^{\prime}\right) \\
\text { otherwise }
\end{array}\right. \\
& \begin{array}{r}
\operatorname{tdepth}\left(\begin{array}{l}
\text { if } P \\
\text { then } S \\
\text { else } S^{\prime} \\
\text { ti } \\
\text { while } P \text { depth } \\
s, \quad S \\
\text { od }
\end{array}\right)=1+\operatorname{tdepth~}(s, S) \\
\text { otherwise }
\end{array}
\end{aligned}
$$

The precondition for $\operatorname{tdepth}(s, S)$ is that $s$ is a statement contained within $S$.

The following is the transformation algorithm.

1. Input program $p$.

2. While $p$ contains one or more exit statements do:

3. Choose an exit statement $s$ of $p$ with greatest tdepth.

4. Let $n$ denote the depth of $s$ in $p$.

5. If $n=0$ then apply Rule 3 to $s$.

6. If $n=1$ then apply Rule 4 or Rule 5 to $s$.

7. If $n>1$ then apply one of Rule 6 , Rule 7 , Rule 8 , or Rule 9 to $s$.

8. Repeatedly apply Rule 2 until there are no instances of exit 0 in $p$.

9. od

10. Output $p$.

\subsection{Proof of correctness}

We first prove a property of the algorithm that will be used to prove that it must terminate.

LEMMA 6.1. Suppose that $s$ is an exit statement in program $S$ and that an application of one of Rule 6 , Rule 7, Rule 8, or Rule 9 in the transformation Algorithm, to an exit statement $s^{\prime} \neq s$, transforms $S$ into $S^{\prime}$. Then $\operatorname{tdepth}(s, S) \geq \operatorname{tdepth}\left(s, S^{\prime}\right)$.

Proof. Assume that the rule being applied is Rule 6 - the other cases follow by a similar argument. Proof by contradiction: suppose that $\operatorname{tdepth}(s, S)<$ $\operatorname{tdepth}\left(s, S^{\prime}\right)$. Recall that Rule 6 applied to exit statement $s^{\prime}$ is: 


\begin{tabular}{|c|}
\hline if $P$ then \\
$S_{1} ;$ \\
if $P^{\prime}$ then \\
$T_{1} ;$ \\
$s^{\prime} ;$ \\
$T_{2}$ \\
else \\
$T_{3}$ \\
fi \\
$S_{2}$ \\
else \\
$S_{3}$ \\
fi
\end{tabular}$\quad \rightarrow \mid$\begin{tabular}{c|} 
if $\left(P \wedge P^{\prime \prime}\right)$ then \\
$S_{1} ;$ \\
$T_{1} ;$ \\
$s^{\prime \prime}$ \\
else \\
if $P$ then \\
$S_{1} ;$ \\
$T_{3} ;$ \\
$S_{2}$ \\
else \\
$S_{3}$ \\
$\mathrm{fi}$ \\
$\mathrm{fi}$
\end{tabular}

If $s^{\prime}$ is exit $n$ then $s^{\prime \prime}$ is the exit statement exit $n-1$. Clearly, this transformation can only increase the value of tdepth for $s$ if $s$ is contained in $S_{1}$. But if $s$ is contained in $S_{1}$ then, since each while loop has at most one exit statement associated with it, $\operatorname{tdepth}(s, S)>$ $\operatorname{tdepth}\left(s^{\prime}, S\right)$. This contradicts the algorithm having been applied to $s^{\prime}$ in $S$.

Proposition 6.13. The transformation algorithm is guaranteed to terminate.

Proof. We need only consider the steps in the loop. It is sufficient to observe that:

1. Each iteration converts a level $n$ exit into a level $n-1$ exit or reduces the value of tdepth for an exit.

2. Any instance of exit 0 generated by this process is removed.

3. Since the algorithm applies a rule to an exit statement of maximum tdepth, no step may introduce a new exit.

4. No step may convert a level $n$ exit into a level $m$ exit for some $m>n$.

5. By Lemma 6.1, no step can increase the value of tdepth of an exit.

Thus each iteration of the loop reduces the sum of the levels of the exit statements plus the sum of tdepth for each exit. The result follows from observing that this value is an integer and is bounded below by 0 .

Proposition 6.14. When applied to a program $p$, the transformation algorithm always terminates with a structured program $p^{\prime}$.

Proof. This follows immediately from the observation that the only constructs that lead to unstructured programs are exit statements and the program cannot terminate if one or more exit statements remain.

THEOREM 6.1. The transformation algorithm is correct.

Proof. By Proposition 6.13, the algorithm must terminate. From Propositions 6.3, 6.5, 6.7, 6.9, and 6.11, we know that the transformation rules preserve branchcoverage equivalence. By Proposition 6.14 we know that the algorithm terminates with a structured program.

Thus, the algorithm must terminate and the result must be a structured program that is branch-coverage equivalent to the original. Thus the algorithm is correct.

By taking a program $p$ and returning a structured branch-coverage equivalent program $p^{\prime}$ we simplify the problem of generating a set of test inputs that satisfies $100 \%$ branch coverage since we can now apply a range of test generation algorithms to $p^{\prime}$. Naturally, having generated a set $T$ of test inputs from $p^{\prime}$ we test $p$ with the elements of $T$.

\subsection{Algorithmic complexity}

This section will prove that the transformation algorithm has low-order polynomial complexity.

THEOREM 6.2. Suppose $p$ has exit statements $s_{1}, \ldots, s_{n}$, exit statement $s_{i}$ is an $n_{i}$ level exit statement and let $d_{i}=\operatorname{tdepth}\left(s_{i}, p\right)$. Let $d=\sum_{i=1}^{n} d_{i}$ and $m=\sum_{i=1}^{n} n_{i}$. The transformation algorithm has time complexity of $O(m+d)$.

Proof. Given exit statement $s_{i}$, by Lemma 6.1, Rules $6,7,8$, and 9 are applied at most $d_{i}$ times to $s_{i}$. Thus Rules $6,7,8$, and 9 are applied at most $d$ times in total.

Rules 3, 4, and 5 reduce the level of one exit and do not increase the level of any exit. Since a rule is applied to an exit statement of maximum tdepth and each loop has at most one exit statement, no step can create a new exit statement. Thus, the number of applications of these three rules is bounded above by $m$.

To conclude, the number of applications of Rules 3 , 4 , and 5 is bounded above by $m$ and the number of applications of Rules $6,7,8$, and 9 is bounded above by $d$. Clearly, the number of applications of Rule 2, which cleans up terms of the form exit 0 , is also bounded above by $m$. The result thus follows.

Note that where we have several procedures or functions, the algorithm may be separately applied to these. Thus, the complexity is linear in the number procedures and functions.

\section{CONCLUSIONS}

Many test-data generation techniques are based upon white-box test criteria. Tools and algorithms that automatically generate test data in order to satisfy a white-box test-criterion are hindered by unstructured control flow. The need for a restructuring transformation to preserve the test-adequacy criterion makes this problem different to the traditional, and well-known, problem of program restructuring transformation. 
This paper uses a novel transformation approach in which the program is restructured in such a way that branch-coverage adequate sets of test inputs are preserved. This form of transformation does not necessarily need to preserve traditional (functional) equivalence, as the transformed program is only required to generate test data. It does, however, need to preserve the sets of branch-coverage adequate test inputs. This new preservation constraint has important theoretical and practical implications and entails proof obligations in terms of branch-coverage adequate sets of test inputs, rather than (for example) functional equivalence.

The approach is illustrated with a set of transformation rules for multiple-level exit statement removal and a simple transformation algorithm for restructuring which uses these rules. The algorithm is proved correct with respect to the requirement that it preserves the branch-coverage adequate sets of test inputs.

Future work will consider the problem of determining, where possible, branch-coverage equivalence. While it is clear that, in general, branch-coverage equivalence is undecidable, there may exist useful conservative decision procedures. The existence of such procedures would simplify the process of verifying transformation rules and might potentially be used to assist in the automated generation of such rules. We also intend to investigate alternative white-box test-criteria such as $100 \%$ statement coverage and $100 \% \mathrm{MC} / \mathrm{DC}$ coverage [41]. We conjecture that notions similar to branchcoverage equivalence will prove useful for many such test criteria.

The transformation algorithm given in this paper was designed to be applied to programs whose predicates (contained in loops that have an exit statement) are side-effect free and in which each loop contains at most one exit statement. There thus remains the problem of finding either a more general transformation algorithm, or transformation rules that preserve branch-coverage equivalence, and transform a program into a program that satisfies these conditions. The novel semantics preserved by branch-coverage preserving transformations suggests the possibility of using slicing to remove parts of the program irrelevant to the satisfaction of a test goal. The incorporation of slicing into our transformation approach is also a topic for future work.

\section{ACKNOWLEDGEMENTS}

We would like to thank the anonymous reviewers for their many helpful comments. In particular, the example used in order to illustrate the restriction, that we have at most one exit statement for a loop, was suggested by one of the reviewers. This work was partially funded by EPSRC grants GR/R43150 (Formal Methods and Testing), GR/R98938 (Testability Transformation), and GR/M78083 (Software Engineering us- ing Metaheuristic INnovative ALgorithms). The work is also supported by two development grants from the DaimlerChrysler Test-Data Generation group, DaimlerChrysler AG Berlin.

\section{REFERENCES}

[1] Boehm, B. W. (1981) Software Engineering Economics. Prentice-Hall, Englewood Cliffs, NJ.

[2] Clarke, L. A. (1976) A system to generate test data and symbolically execute programs. IEEE Transactions on Software Engineering, 2, 215-222.

[3] Goldberg, A., Wang, T. C., and Zimmerman, D. (1994) Applications of feasible path analysis to program testing. In Ostrand, T. (ed.), Proceedings of the 1994 International Symposium on Software Testing and Analysis (ISSTA), Seattle, WA, USA, pp. 80-94. ACM Press, New York.

[4] King, J. C. (1976) Symbolic execution and program testing. Communications of the ACM, 19, 385-394.

[5] DeMillo, R. A. and Offutt, A. J. (1993) Experimental results from an automatic test generator. $A C M$ Transactions of Software Engineering and Methodology, 2, 109-127.

[6] Offutt, A. J. (1990) An integrated system for automatically generating test data. In $\mathrm{Ng}$, Peter A.; Ramamoorthy, C.V.; Seifert, Laurence C.; Yeh, R. T. (ed.), Proceedings of the First International Conference on Systems Integration, Morristown, NJ, April, pp. 694-701. IEEE Computer Society Press, Los Alamitos, California, USA.

[7] Ferguson, R. and Korel, B. (1996) The chaining approach for software test data generation. ACM Transactions on Software Engineering and Methodology, 5, 63-86.

[8] Jones, B., Sthamer, H.-H., and Eyres, D. (1996) Automatic structural testing using genetic algorithms. The Software Engineering Journal, 11, 299-306.

[9] Michael, C., McGraw, G., and Schatz, M. (2001) Generating software test data by evolution. IEEE Transactions on Software Engineering, 27, 1085-1110.

[10] Mueller, F. and Wegener, J. (1998) A comparison of static analysis and evolutionary testing for the verification of timing constraints. 4th IEEE RealTime Technology and Applications Symposium (RTAS '98), Washington - Brussels - Tokyo, June, pp. 144154. IEEE Computer Society Press, Los Alamitos, California, USA.

[11] Pargas, R. P., Harrold, M. J., and Peck, R. R. (1999) Test-data generation using genetic algorithms. The Journal of Software Testing, Verification and Reliability, 9, 263-282.

[12] Pohlheim, H. and Wegener, J. (1999) Testing the temporal behavior of real-time software modules using extended evolutionary algorithms. In Banzhaf, W., Daida, J., Eiben, A. E., Garzon, M. H., Honavar, V., Jakiela, M., and Smith, R. E. (eds.), Proceedings of the Genetic and Evolutionary Computation Conference, Orlando, Florida, USA, 13-17 July 1795. Morgan Kaufmann, San Francisco, CA 94104, USA.

[13] Schultz, A., Grefenstette, J., and Jong, K. (1993) Test and evaluation by genetic algorithms. IEEE Expert, 8, $9-14$. 
[14] Tracey, N., Clark, J., Mander, K., and McDermid, J. (2000) Automated test-data generation for exception conditions. Software Practice and Experience, 30, 6179 .

[15] DeMillo, R. and Offutt, A. J. (1991) Constraint-based automatic test data generation. IEEE Transactions on Software Engineering, 17, 900-910.

[16] Ferrante, J., Ottenstein, K. J., and Warren, J. D. (1987) The program dependence graph and its use in optimization. ACM Transactions on Programming Languages and Systems, 3, 319-349.

[17] Agrawal, H. (1994) On slicing programs with jump statements. ACM SIGPLAN Conference on Programming Language Design and Implementation, Orlando, Florida, June 20-24, pp. 302-312. ACM Press, New York, NY. Proceedings in SIGPLAN Notices, 29(6), June 1994.

[18] Ball, T. and Horwitz, S. (1993) Slicing programs with arbitrary control-flow. In Fritzson, P. (ed.), $1^{\text {st }}$ Conference on Automated Algorithmic Debugging, Linköping, Sweden, pp. 206-222. Springer-Verlag, Heidelberg, LNCS volume 749.

[19] Choi, J. and Ferrante, J. (1994) Static slicing in the presence of goto statements. ACM Transactions on Programming Languages and Systems, 16, 1097-1113.

[20] Sinha, S., Harrold, M. J., and Rothermel, G. (1999) System-dependence-graph-based slicing of programs with arbitrary interprocedural control-flow. Proceedings of the 21st International Conference on Software Engineering, Melbourne, Australia, May, pp. 432-441. ACM Press, New York, NY.

[21] Ramshaw, L. (1988) Eliminating goto's while preserving program structure. Journal of the ACM, 35, 893920.

[22] Erosa, A. M. and J.Hendren, L. (1994) Taming Control Flow: A Structured Approach to Eliminating GOTO Statements. IEEE International Conference on Computer Languages, Toulouse, France, pp. 229240. IEEE Computer Society Press, Los Alamitos, California, USA.

[23] Harman, M., Hu, L., Hierons, R. M., Wegener, J., Sthamer, H., Baresel, A., and Roper, M. (2004) Testability transformation. IEEE Transactions on Software Engineering, 30, 3-16.

[24] Ward, M. (1999) Assembler to C migration using the FermaT transformation system. IEEE International Conference on Software Maintenance (ICSM'99), Oxford, UK, August, pp. 67-76. IEEE Computer Society Press, Los Alamitos, California, USA.

[25] Jones, B. F., Eyres, D. E., and Sthamer, H.-H. (1998) A strategy for using genetic algorithms to automate branch and fault-based testing. The Computer Journal, 41, 98-107.

[26] Tracey, N., Clark, J., and Mander, K. (1998) Automated program flaw finding using simulated annealing. International Symposium on Software Testing and Analysis, Clearwater Beach, Florida, USA, March, pp. 73-81. ACM Press, New York, NY.

[27] Wegener, J., Sthamer, H., Jones, B. F., and Eyres, D. E. (1997) Testing real-time systems using genetic algorithms. Software Quality, 6, 127-135.
[28] Voas, J. M. and Miller, K. W. (1995) Software testability: The new verification. IEEE Software, 12, $17-28$.

[29] Böhm, C. and Jacopini, G. (1966) Flow diagrams, Turing machines, and languages with only two formation rules. Communications of the ACM, 9, 366-372. Presented as an invited talk at the 1964 International Colloquium on Algebraic Linguistics and Automata Theory.

[30] Cooper, D. (1967) Böhm and Jacopini's reduction of flow charts. Communications of the ACM, 10, 463473.

[31] Dijkstra, E. W. (1972) A discipline of programming,. Prentice-Hall, Englewood Cliffs, NJ.

[32] Harel, D. (1980) On folk theorems. Communications of the ACM, 23, 379-389.

[33] Kosaraju, S. R. (1974) Analysis of structured programs. Journal of Computer and System Sciences, 9, 232-255.

[34] Peterson, W. W., Kasami, T., and Tokura, N. (1973) On the capabilities of while, repeat, and exit statements. Communications of the ACM, 16, 503512.

[35] Ward, M. Reverse engineering through formal transformation. The Computer Journal, 37, 795-813.

[36] Knuth, D. E. and Floyd, R. W. (1971) Notes on avoiding "go to" statements. Information Processing Letters, 1, 23-31.

[37] Dolado, J. J., Harman, M., Otero, M. C., and Hu, L. (2003) An empirical investigation of the influence of a type of side effects on program comprehension. IEEE Transactions on Software Engineering, 29, 665-670.

[38] Harman, M., Hu, L., Zhang, X., and Munro, M. (2001) Side-effect removal transformation. $9^{\text {th }}$ IEEE International Workshop on Program Comprehension (IWPC'01), Toronto, Canada, May, pp. 310-319. IEEE Computer Society Press, Los Alamitos, California, USA.

[39] Aho, A. V., Sethi, R., and Ullman, J. D. (1986) Compilers: Principles, techniques and tools. Addison Wesley, Reading, Massachusetts.

[40] Sivagurunathan, Y., Harman, M., and Danicic, S. (1997) Slicing, I/O and the implicit state. In Kamkar, M. (ed.), $3^{\text {rd }}$ International Workshop on Automated Debugging ( $A A D E B U G^{\prime} 9^{\prime 7}$ ), Linköping, Sweden, May, Linköping Electronic Articles in Computer and Information Science, 2, pp. 59-65.

[41] RTCA/DO-178B (1992) Software Considerations in Airborne Systems and Equipment Certification. RTA, Washington, DC. 